\title{
Características de la mortalidad por enfermedad isquémica del corazón en Costa Rica de 1970 al 2014
}

\author{
Characteristics of mortality due to ischemic heart disease in Costa Rica from 1970 to 2014 \\ Ronald Evans-Meza ${ }^{1 *}$, José Pérez-Fallas ${ }^{2}$ y Roger Bonilla-Carrión ${ }^{2}$ \\ ${ }^{1}$ Unidad de Investigación e Innovación en Ciencias de la Salud; ${ }^{2}$ Departamento de Estadística, Unidad de Investigación e Innovación en Ciencias \\ de la Salud, Universidad Hispanoamericana de San José, Costa Rica
}

\begin{abstract}
Resumen
Objetivo: Actualizar algunos aspectos importantes de la epidemiología descriptiva de la enfermedad isquémica del corazón (EIC) en Costa Rica durante el periodo 1970-2014. Métodos: Se obtuvieron las tasas de mortalidad por EIC en dos periodos. De 1930 al año 1969 y luego, para un análisis más específico, de 1970 al 2014, utilizando promedios móviles y quinquenios, a partir de datos del Instituto Nacional de Estadística y del Centro Centroamericano de Población, para analizarlos en relación con edad, sexo, ubicación geográfica y aporte porcentual a la mortalidad general. También se obtuvo información para determinar la carga de la enfermedad, en este caso mediante años de vida ajustados por discapacidad, años de vida perdidos y años de vida perdidos por discapacidad. Resultados: Se apreció ascenso de las tasas en la población general y con mayor empuje en la población de 35 a 74 años, hasta el quinquenio 1995-1999, a partir del cual se inicia un descenso que aparentemente se detiene durante el quinquenio 2010-2014 (ambos sexos: $r=0.9964, r^{2}=0.9928, \beta=-2.4950$, $p=0.04$; hombres: $r=0.9994, r^{2}=0.9988, \beta=-2.770, p=0.02$; mujeres: $r=0.9896, r^{2}=0.9793, \beta=-2.4950, p=0.07$ ). Las tasas más altas se presentan en las provincias del centro del país. Predomina durante todo el periodo en el sexo masculino y representa en promedio el $14 \%$ de la mortalidad general. Conclusión: La mortalidad por EIC está en fase de disminución, pero el aumento de la obesidad y el sobrepeso en el país amenaza con frenar dicha tendencia, al menos durante el último quinquenio.
\end{abstract}

Palabras claves: Mortalidad. Enfermedad isquémica del corazón. Estudios descriptivos. Epidemiología descriptiva. Costa Rica.

\begin{abstract}
Objective: To update some important aspects of the descriptive epidemiology of ischemic heart (EIC) disease in Costa Rica during the period 1970-2014. Methods: EIC death rates were obtained in two periods: from 1930 to 1969 and then, for a more specific analysis, from 1970 to 2014, using moving and five-year averages, based on data from the Instituto Nacional
\end{abstract}

\footnotetext{
Correspondencia:

*Ronald Evans-Meza

Universidad Hispanoamericana

Barrio Aranjuez, de la esquina noreste del

Hospital Calderón Guardia, 100 metros este,

300 metros Norte

Fecha de recepción: 07-06-2018

Fecha de aceptación: 28-11-2018

E-mail: roevansme@gmail.com

DOI: 10.24875/ACM.M19000014

Disponible en internet: 19-03-2019 Arch Cardiol Mex. 2019;89(1):38-50 www.archivoscardiologia.com 1405-9940 @ 2019 Instituto Nacional de Cardiología Ignacio Chávez. Publicado por Permanyer México SA de CV. Este es un artículo Open Access bajo la licencia CC BY-NC-ND (http://creativecommons.org/licenses/by-nc-nd/4.0/).
} 
de Estadística and the Centro Centroamericano de Población, to analyze them according to age, sex, geographical location and percentage contribution to the general mortality. Information was also obtained to determine the burden of the disease, in this case through Years of Adjusted Life for Disability, Years of Life Lost and Years of Life lost due to Disability. Results: There was a rise in rates in the general population, with greater momentum in the population from 35 to 74 years, up to the five-year period 1995-1999, after which a decline began that apparently stopped during the five-year period 2010-2014 (both sexes: $r=0.9964, r^{2}=0.9928, \beta=-2.4950, p$ 0.04; men: $r=0.9994, r^{2}=0.9988, \beta=-2.770, p=0.02 ;$ women: $r=0.9896, r^{2}$ $=0.9793, \beta=-2.4950, p=0.07$ ). The highest rates occur in the provinces of the center of the country. It predominates during the whole period in the male sex and represents on average $14 \%$ of the general mortality. Conclusions: Mortality due to EIC is in a phase of decline but the increase in obesity and overweight in the country threatens to slow down this trend, at least during the last five years.

Key words: Mortality. Ischemic heart disease. Descriptive studies. Descriptive epidemiology. Costa Rica.

\section{Introducción}

Desde hace ya hace varias décadas se sabe que las enfermedades del aparato circulatorio(enfermedades cardiovasculares [ECV]), entendiendo como tal a los eventos mortales incluidos en la X Clasificación Internacional de Enfermedades bajo los códigos 100-199, Q20 y Q28 cuando estos dos últimos están disponibles, son la primera causa mundial de muerte y, entre ellas, la enfermedad isquémica del corazón (EIC) ocupa el primer lugar, incluso en los países más pobres de la tierra. En el año 2013 hubo 17.3 millones de defunciones por ECV, lo que representa el $31 \%$ de todas las muertes en el mundo y aproximadamente el $50 \%$ del total de las enfermedades crónicas no transmisibles. Para el 2030 se estima que el número de muertes por estas causas ascienda a 23.6 millones ${ }^{1}$. Afortunadamente, sin embargo, las tasas están disminuyendo. Así, por ejemplo, en el 2005 la tasa fue 338.1 por 100.000 , pero en el 2015 bajó a 285.5 por 100.000 , lo que significó una disminución del $15.6 \%$.

En el año 2010, las ECV ocasionaron en el mundo la pérdida de 293 millones de años de vida perdidos ajustados por discapacidad (AVAD o DALYs, por sus siglas en inglés) ${ }^{3}$. Globalmente, las ECV fueron la primera causa de años de vida perdidos (YLLs, por sus siglas en inglés) ${ }^{4}$.

Para evitar confusiones, de aquí en adelante se considerará sinónimos a las ECV y las enfermedades del aparato circulatorio.

Mundialmente existen grandes diferencias en las tasas estandarizadas de mortalidad por enfermedades del aparato circulatorio. Varios países tienen tasas para varones superiores a mil por cien mil habitantes, como Rusia $(1,087.9)$, Ucrania $(2,012.0)$ y Bielorrusia $(1,178.0)$, a los que siguen con tasas un poco menores Bulgaria (783.3), Rumania (594.6) y Hungría (499.7). En general, las tasas más elevadas corresponden en Europa a los países del este, antiguamente bajo regímenes comunistas ${ }^{1}$.

En los EE.UU. desde el año 1900 (con excepción del 1918, año de la gran pandemia de gripe) las ECV han ocasionado más muertes que cualquier otra causa importante de mortalidad. En el año 2013 fueron la causa del $30.8 \%$ del total de defunciones, lo que equivale más o menos a una de cada tres muertes; cada 40 segundos en promedio fallece un estadounidense por ECV. Se ha estimado que si todas las principales causas de ECV fuesen eliminadas, la expectativa de vida podría incrementarse en siete años ${ }^{1}$.

La EIC es la principal causa de muerte de todas las ECV y su tasa estandarizada ha venido disminuyendo en casi todas las regiones del mundo en los últimos quinquenios, pero su carga de enfermedad global se ha incrementado en un $29 \%$, debido principalmente al aumento poblacional y al incremento de una población cada vez más longeva. En casi todos los países desarrollados la reducción supera el 50\% desde 1990; en Dinamarca, Holanda, Noruega e Israel incluso alcanzó el $70 \%{ }^{5}$. En las Américas, las ECV igualmente han venido ocupando los primeros puestos de muertes desde hace ya varias décadas, sobresaliendo la EIC en la primera posición. En la tabla 1 aparecen las tasas por EIC en relación con el sexo, para una fecha relativamente reciente ${ }^{6}$.

Costa Rica es un país pequeño, ya que tiene una extensión territorial de $51.100 \mathrm{~km}^{2}$ y una población de cinco millones de habitantes (octubre de 2018). Como se aprecia en el mapa (Fig. 1), está dividido en siete provincias, por el norte limita con Nicaragua y por el sur con Panamá. En Costa Rica, al igual que lo que ocurre prácticamente en todo el mundo, las enfermedades crónicas no transmisibles vienen siendo desde hace ya varias décadas las principales causas de muerte en el país, tanto que constituyen el $79 \%$ de todas ellas ${ }^{7}$.Algo semejante ocurre en todo el mundo, ya que dichos 
Tabla 1. Tasa de mortalidad por enfermedades isquémicas del corazón en América para el año 2015. Tasas por 100.000 habitantes

\begin{tabular}{|c|c|c|c|}
\hline Regiones y países & Total & Hombres & Mujeres \\
\hline Las Américas & 63.1 & 82.8 & 46.6 \\
\hline América del norte & 61.6 & 83.1 & 43.3 \\
\hline Bermuda & 54.9 & 66.0 & 44.3 \\
\hline \multicolumn{4}{|l|}{ Canadá } \\
\hline EE.UU. & 61.6 & 83.1 & 43.3 \\
\hline América Latina y el caribe & 63.7 & 82.4 & 48.2 \\
\hline América Latina & 63.8 & 82.5 & 48.3 \\
\hline México & 74.9 & 94.0 & 58.3 \\
\hline Istmo centroamericano & 63.7 & 75.2 & 54.0 \\
\hline Belice & 55.5 & 81.7 & 30.4 \\
\hline Costa Rica & 57.6 & 75.5 & 41.6 \\
\hline El Salvador & 55.8 & 64.4 & 48.9 \\
\hline Guatemala & 65.7 & 77.3 & 56.1 \\
\hline Honduras & 48.2 & 51.2 & 44.9 \\
\hline Nicaragua & 95.5 & 111.4 & 82.5 \\
\hline Panamá & 59.1 & 75.4 & 44.2 \\
\hline Caribe latino & 88.9 & 106.0 & 73.4 \\
\hline Cuba & 80.7 & 95.5 & 66.9 \\
\hline Guadalupe & 11.9 & 14.7 & 9.5 \\
\hline Guayana francesa & 8.8 & 9.3 & 7.6 \\
\hline \multicolumn{4}{|l|}{ Haití } \\
\hline Martinica & 10.5 & 16.1 & 6.2 \\
\hline Puerto Rico & 49.0 & 67.8 & 34.8 \\
\hline República dominicana & 119.9 & 139.3 & 101.4 \\
\hline Área andina & 77.7 & 100.0 & 59.0 \\
\hline \multicolumn{4}{|l|}{ Bolivia } \\
\hline Colombia & 102.6 & 126.3 & 83.2 \\
\hline Ecuador & 46.5 & 61.3 & 33.1 \\
\hline Perú & 29.1 & 38.2 & 21.1 \\
\hline Venezuela & 104.3 & 142.8 & 72.6 \\
\hline Brasil & 54.4 & 72.7 & 39.6 \\
\hline Cono sur & 37.6 & 54.8 & 24.1 \\
\hline Argentina & 34.7 & 52.3 & 21.4 \\
\hline Chile & 33.7 & 49.4 & 21.0 \\
\hline Paraguay & 67.5 & 83.9 & 52.0 \\
\hline Uruguay & 36.4 & 55.4 & 23.1 \\
\hline
\end{tabular}

Adaptada de Organización Panamericana de la Salud, $2016^{6}$. padecimientos fueron la causa en el 2016 del $72.3 \%$ de todas las defunciones (intervalo de confianza del 95\% [IC 95\%]: 71.2-73.2) ${ }^{8}$. Dentro de este gran grupo, las ECV se han convertido en la primera causa de muerte desde hace varias décadas. En el año 2015, en Costa Rica ocasionaron 5,689 defunciones, con una tasa de 98.07 por 100,000 habitantes. Entre todas ellas, la EIC siempre ha ocupado el primer lugar y como causa específica aparece en primer término el infarto al miocardio, mientras que como segunda causa de muerte por ECV aparecen las enfermedades cerebrovasculares. La EIC fue la causa de 2,690 defunciones, con una tasa de 47.93 por 100,000 , lo que equivale al $47.3 \%$ del grupo. Mientras tanto el infarto agudo al miocardio (IAM), que es la principal causa de muerte entre el segmento de la EIC, presentó 1,383 defunciones, con una tasa de 28.6 por 100,000 , equivalente al $51.2 \%$ de las muertes de dicho segmento, siempre para el mismo año $2015^{9}$.

En Costa Rica se han publicado pocos trabajos sobre epidemiología descriptiva de la EIC. Roselló y Guzmán ${ }^{10}$ analizaron la mortalidad durante el periodo 1970-2002 según su distribución geográfica y lugar de ocurrencia. Posteriormente estas dos investigadoras publicaron otro artículo sobre el mismo tema, en este caso con mayor extensión y profundidad, ya que incluyeron además la evolución de la mortalidad por ECV, la EIC y el IAM durante el periodo 1970-2001'11.

De allí la importancia de actualizar y ampliar la información existente al respecto, propósito principal de nuestra colaboración.

El objetivo de este trabajo consiste en describir y actualizar algunos aspectos de la epidemiología descriptiva de la EIC, especialmente de su mortalidad, durante los años comprendidos entre 1970 y 2014.

\section{Métodos}

Inicialmente se obtuvieron las cifras de muertes para todas las edades por EIC en Costa Rica desde 1930 a 1969 de los anuarios de estadísticas del Instituto Nacional de Estadística (INEC) ${ }^{12}$, y posteriormente de la base de datos del Centro Centroamericano de Población (CCP) de la Universidad de Costa Rica a partir de 1970 hasta el año $2014^{13}$. De esas mismas fuentes se obtuvo la población total del país para los mismos años del periodo estudiado, con el fin de calcular las tasas brutas "suavizadas" por promedios móviles trienales y también por quinquenios. Los promedios móviles se utilizan en este sentido ${ }^{14,15}$, para lo cual se escogió tres años cada vez: para comenzar se suman las tres primeras tasas y se dividen entre tres, colocando la tasa 


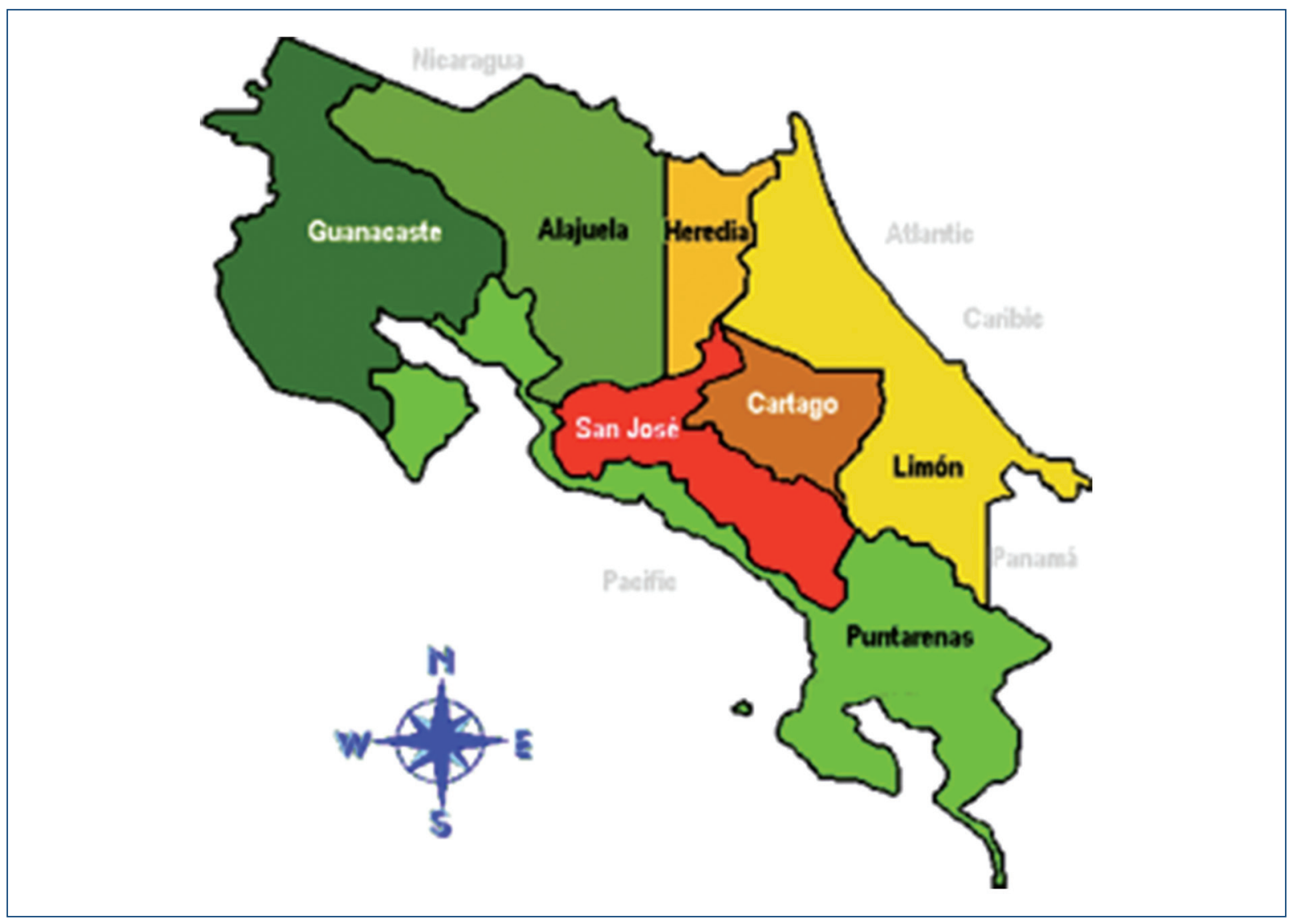

Figura 1. Mapa de Costa Rica (adaptado de Caja Costarricense de Seguro Social, 2014).12

frente al año intermedio de ese primer trienio, luego los totales sucesivos se obtendrán excluyendo del total precedente el primer año del trienio y añadiéndole el siguiente año hasta completar la serie.

Posteriormente se obtuvieron las muertes totales por dichas causas (I20 - I25 CIE 10), clasificadas por sexo, de la población de 35 a 74 años para el periodo comprendido entre el 1 de enero de 1970 y el 31 de diciembre del año 2014. Con la población de ese grupo etario se calcularon las tasas estandarizadas respectivas, dado que es el grupo de edad con mayor riesgo de morir por tales enfermedades y también para poder realizar comparaciones internacionales. Como población estándar se tomó la de América Latina.

Para evaluar las tendencias o cambios seculares de las tasas de mortalidad por EIC se utilizó en primer término las modificaciones porcentuales de estas y en segundo lugar las técnicas de regresión, obteniendo los coeficientes de correlación o determinación $\left(r^{2}\right)$, la estimación de la beta y por último, el grado de significación ( $p$ ) para determinar si los cambios fueron o no significativos estadísticamente.
También se calcularon las tasas brutas y tipificadas de mortalidad por cien mil habitantes por provincias y regiones de Costa Rica. La estandarización igualmente se hizo utilizando la distribución por edad de la población de América Latina 2015 (Centro Latinoamericano y Caribeño de Demografía [CELADE] $)^{16}$. Esta población estándar la hemos utilizado en otros trabajos, por permitirnos la mejor comparación de tasas con las que tienen los demás países latinoamericanos.

Para la obtención de las defunciones por EIC se utilizó la octava Clasificación Internacional de Enfermedades (CIE), específicamente los códigos 410, para los años 1970-79, los códigos 410 de la CIE-9 para el periodo 19801996 y los códigos 121 de la CIE-10 para el lapso entre 1997-2014. Esta última distingue las siguientes entidades: - Enfermedades isquémicas del corazón (I20 a I25)

- I20 Angina de pecho.

- I21 Infarto agudo de miocardio con elevación de ST (IAMCEST) y sin elevación de ST (IAMSEST).

- 122 Infarto agudo de miocardio subsiguiente con elevación de ST (IAMCEST) (IMEST) (STEMI) y sin elevación de ST (IAMSEST) (IMNEST) (NSTEMI). 
- I23 Complicaciones en curso, tras infarto de miocardio con elevación de ST (IAMCEST) (IMEST) (STEMI) y sin elevación del ST (IAMSEST) (IMSEST) (NSTEMI) (dentro del periodo de 28 días).

- 124 Otras enfermedades isquémicas agudas cardíacas.

- I25 Enfermedad isquémica crónica cardíaca.

Con respecto al cambio de los códigos de la CIE-9 a la CIE-10 comprobamos que las tasas no variaron prácticamente entre 1990 y 1999, década a mitad de la cual se produjo el cambio entre ambas clasificaciones. Por otra parte, el aporte porcentual de la mortalidad por EIC a la mortalidad general tampoco varió entre los años inmediatos antes y después del cambio de CIE (se mantuvo entre 14.41 y 14.24\%). En el ámbito internacional, en España no constituyó motivo de preocupación los cambios ocurridos entre los criterios de la CIE-9 a la CIE-10, ya que estos se han mantenido relativamente estables ${ }^{17}$.

En el caso de la mortalidad anterior al año 1970 se trabajó con las CIE vigentes, tal como aparecieron en los anuarios de estadística del INEC.Para la tabulación y análisis de los datos, se utilizó el programa STATA ${ }^{\circledR 18}$.

Para omitir el problema de la clasificación inapropiada, que ha sido estudiado por la Oragnización Mundial de la Salud ${ }^{19}$, se determinó el porcentaje de las patologías mal definidas, muchas de las cuales en propiedad corresponden a EIC, como la taquicardia ventricular paroxística, la fibrilación ventricular, el paro cardíaco, la insuficiencia cardíaca y la insuficiencia cardíaca no especificada, entre otras, para dos periodos específicos. El primero de 1980 a 1999 y el segundo del 2000 al 2016. En el primer caso hubo una disminución persistente del porcentaje de ellas sobre el total de enfermedades del corazón, variando desde un máximo de $27.1 \%$ en el año 1980 a $10.19 \%$ en 1995 . En el caso de los años que corresponden a la CIE-10, el porcentaje promedio de mal definidas fue de 4.30 , con valores extremos de $4.94 \%$ (año 2001) y $3.13 \%$ (año 2008). De acuerdo con estos bajos porcentajes, Costa Rica está en el grupo de países considerados como de «baja mal codificación», por tanto es posible asumir que no han afectado significativamente las tasas de la EIC.

\section{Resultados}

En la tabla 2 aparece la evolución de los promedios móviles de las tasas brutas de mortalidad por EIC en Costa Rica de 1930 al 2014. En términos generales se observa un ascenso continuado hasta el año 2004, a partir del cual las tasas tienden a estabilizarse hasta el 2009. A partir de ese año, las tasas más bien muestran una moderada elevación.

En la tabla 3 se muestra la anterior información, pero esta vez tomando en cuenta los promedios quinquenales y su relación porcentual con la mortalidad general. Igual tendencia se observa cuando se estudian las tasas promedio por quinquenios en el mismo lapso de tiempo, pero el descenso hasta el quinquenio 2004-2009 se interrumpe, ya que le sigue un ligero incremento en el siguiente periodo, algo semejante a lo observado en la tabla 2.

El aporte porcentual de la mortalidad por EIC con respecto a la mortalidad general fue prácticamente insignificante durante el periodo 1930-1954 (menos de un 1\%), pero en el lapso de tiempo comprendido entre el quinquenio 1955-1959 y el 1975-1979, el incremento es notorio, en especial a partir de 1980, cuando el porcentaje alcanza los dos dígitos hasta estabilizarse en un $14 \%$.

También se analizaron las tasas promedio quinquenales ajustadas para el grupo etario de 35 a 74 años entre los años 1970-1974 y 2010-2014. A simple vista se aprecia que las tasas por cien mil habitantes se incrementaron hasta el lustro 1995-1999, a partir del cual se visualiza un descenso importante y sostenido en los tres quinquenios subsiguientes (Tabla 4), tanto en hombres como en mujeres. El descenso porcentual entre los quinquenios extremos para las mujeres fue de 44.19, mientras que para los hombres fue de 13.16. En este caso llama la atención la enorme diferencia del descenso a favor de las mujeres. Para ambos sexos el descenso correspondió a un $25.88 \%$.

Cuando dicho análisis se realiza de acuerdo al sexo (Tablas 4 y 5, Fig. 2) al separar la serie en dos periodos (1970-1999 y 2000-2014) se aprecia que durante el primer periodo no hubo disminución alguna de las tasas (salvo en los varones, $p=0.05$ ) y más bien se incrementaron en las mujeres y en la tasa total, pero ocurrió lo inverso durante el periodo 2000-2014, dado que para los varones se aprecia una tendencia significativa al descenso $\left(r=0.994, r^{2}=0.9884, \beta=-2.7270, p=0.02\right)$ y lo mismo ocurrió cuando se tuvieron en cuenta los dos sexos $\left(r=0.9964, r^{2}=0.9928, \beta=-2.4950, p=0.04\right)$. Para las mujeres las tasas también disminuyeron, pero la tendencia no fue significativa por poco margen $(p=0.07)$.

\section{Otros indicadores de la carga de la enfermedad}

Asimismo, se obtuvieron otros indicadores, en este caso extraídos de la página del Instituto de Métricas y Evaluación de Salud (IHME) de la Universidad de 
Tabla 2. Promedios móviles de las tasas brutas de mortalidad por cardiopatía isquémica en Costa Rica, 1930-2014

\begin{tabular}{|c|c|c|c|}
\hline Año & Promedios móviles & Año & Promedios móviles \\
\hline 1930 & 1.76 & 1973 & 37.00 \\
\hline 1931 & 1.88 & 1974 & 38.16 \\
\hline 1932 & 1.86 & 1975 & 37.84 \\
\hline 1933 & 1.68 & 1976 & 35.56 \\
\hline 1934 & 1.76 & 1977 & 36.52 \\
\hline 1935 & 2.09 & 1978 & 39.02 \\
\hline 1936 & 2.28 & 1979 & 42.68 \\
\hline 1937 & 2.50 & 1980 & 44.33 \\
\hline 1938 & 2.95 & 1981 & 46.10 \\
\hline 1939 & 2.94 & 1982 & 48.63 \\
\hline 1940 & 3.82 & 1983 & 51.91 \\
\hline 1941 & 3.64 & 1984 & 55.32 \\
\hline 1942 & 3.89 & 1985 & 55.96 \\
\hline 1943 & 2.65 & 1986 & 55.09 \\
\hline 1944 & 3.05 & 1987 & 53.64 \\
\hline 1945 & 3.01 & 1988 & 54.28 \\
\hline 1946 & 3.28 & 1989 & 55.08 \\
\hline 1947 & 2.56 & 1990 & 54.43 \\
\hline 1948 & 2.43 & 1991 & 53.80 \\
\hline 1949 & 2.65 & 1992 & 55.17 \\
\hline 1950 & 2.86 & 1993 & 56.95 \\
\hline 1951 & 5.12 & 1994 & 58.30 \\
\hline 1952 & 8.44 & 1995 & 58.88 \\
\hline 1953 & 11.84 & 1996 & 57.74 \\
\hline 1954 & 13.18 & 1997 & 56.47 \\
\hline 1955 & 13.55 & 1998 & 56.12 \\
\hline 1956 & 13.79 & 1999 & 57.68 \\
\hline 1957 & 13.62 & 2000 & 59.21 \\
\hline 1958 & 14.07 & 2001 & 58.97 \\
\hline 1959 & 15.14 & 2002 & 57.53 \\
\hline 1960 & 15.82 & 2003 & 55.72 \\
\hline 1961 & 16.67 & 2004 & 55.81 \\
\hline 1962 & 17.43 & 2005 & 54.52 \\
\hline 1963 & 20.81 & 2006 & 54.62 \\
\hline 1964 & 22.94 & 2007 & 54.47 \\
\hline
\end{tabular}

(Continúa)
Tabla 2. Promedios móviles de las tasas brutas de mortalidad por cardiopatía isquémica en Costa Rica, 1930-2014 (Continuación)

\begin{tabular}{|l|c|c|c|}
\hline Año & Promedios móviles & Año & Promedios móviles \\
\hline 1965 & 26.67 & 2008 & 54.73 \\
\hline 1966 & 28.44 & 2009 & 54.84 \\
\hline 1967 & 33.25 & 2010 & 56.04 \\
\hline 1968 & 36.87 & 2011 & 56.33 \\
\hline 1969 & 37.02 & 2012 & 56.85 \\
\hline 1970 & 34.19 & 2013 & 57.29 \\
\hline 1971 & 31.50 & 2014 & 60.07 \\
\hline 1972 & 33.64 & & \\
\hline
\end{tabular}

Elaboración propia con datos de anuarios estadísticos y del Centro Centroamericano de Población

Washington, Seattle ${ }^{20}$, con el fin de complementar la carga de la enfermedad por CIC en Costa Rica. En primer término, los Años Vida Ajustados por Discapacidad durante el periodo 1990-2014, tal cual se aprecia en la figura 3. Se observa un descenso continuo hasta el año 2009 y luego la tendencia se estabiliza. Cuantitativamente los AVAD entre los años 1990 y 2014 disminuyeron de 2505 (IC 95\%: 2,378.99-2,642.33) a 1,319.57 (IC 95\%: 1,233.86-1,421.17), para un descenso porcentual del $47.3 \%$

En la figura 4 se nota la evolución de los años de vida potencialmente perdidos (AVP), también durante el mismo periodo de 1990 al 2014, apreciándose, igual que en la figura anterior, un descenso durante este lapso de tiempo y una estabilización de las tasas en los últimos cinco años. En números, durante el primero de los años anteriormente citados se obtuvo 2,401 por cien mil habitantes (IC 95\%: 2,286.98-1,533.93), disminuyendo a 1,214.93 (IC 95\%: 1,129.17-1,311.83), para un descenso porcentual del $50.14 \%$.

En cuanto a años de vida con discapacidad (AVD), en la figura 5 se nota un ascenso de las tasas durante la década inicial para luego estabilizarse hasta los años finales del estudio. Los AVD entre 1990 y 2014 pasaron de 103.41 por cien mil habitantes (IC 95\%: 71.01-147.14) a 104.75 (IC 95\%: 73.70-147.14); es decir, permanecieron prácticamente iguales (incremento del $0.1 \%$ ).

\section{Distribución geográfica}

Las tasas brutas más elevadas durante los cinco primeros quinquenios correspondieron a las provincias de $\mathrm{He}-$ redia, San José y Cartago. Luego Heredia cedió el puesto 
Tabla 3. Tasas brutas de mortalidad por cardiopatía isquémica en Costa Rica, 1930-2014. Promedios quinquenales y relación porcentual con la mortalidad general

\begin{tabular}{|c|c|c|c|c|}
\hline Quinquenio & N. ${ }^{0}$ muertes promedio & Tasa promedio & Cambio porcentual & Relación porcentual \\
\hline 1930-1934 & 9 & 1.7 & & 0.08 \\
\hline 1935-1939 & 15 & 2.6 & 52.92 & 0.13 \\
\hline $1940-1944$ & 21 & 3.2 & 22.86 & 0.18 \\
\hline 1945-1949 & 21 & 2.9 & -10.40 & 0.21 \\
\hline 1950-1954 & 79 & 8.3 & 187.32 & 0.80 \\
\hline $1955-1959$ & 157 & 14.0 & 69.27 & 1.58 \\
\hline 1960-1964 & 257 & 18.8 & 33.96 & 2.36 \\
\hline $1965-1969$ & 537 & 33.1 & 75.73 & 4.76 \\
\hline 1970-1974 & 645 & 34.5 & 4.43 & 6.10 \\
\hline 1975-1979 & 808 & 38.0 & 9.92 & 8.96 \\
\hline 1980-1984 & 1197 & 48.9 & 28.93 & 12.69 \\
\hline 1985-1989 & 1550 & 55.4 & 13.09 & 14.46 \\
\hline 1990-1994 & 1766 & 55.6 & 0.37 & 14.41 \\
\hline 1995-1999 & 2067 & 57.3 & 3.12 & 14.24 \\
\hline $2000-2004$ & 2298 & 57.3 & -0.07 & 14.76 \\
\hline $2005-2009$ & 2256 & 52.0 & -9.19 & 13.27 \\
\hline 2010-2014 & 2726 & 58.58 & -0.12 & 14.01 \\
\hline
\end{tabular}

Elaboración propia con datos de anuarios estadísticos y del Centro Centroamericano de Población

Tabla 4. Mortalidad por cardiopatía isquémica en personas entre 35 y 74 años por quinquenios en Costa Rica, 1970-2014. Tasas ajustadas por 100.000 habitantes

\begin{tabular}{|l|c|c|c|}
\hline Quinquenio & Mujeres & Hombres & Total \\
\hline $1970-1974$ & 68.83 & 98.93 & 83.88 \\
\hline $1975-1979$ & 61.15 & 108.55 & 84.85 \\
\hline $1980-1984$ & 74.32 & 123.88 & 99.10 \\
\hline $1985-1989$ & 72.61 & 134.07 & 103.34 \\
\hline $1990-1994$ & 69.92 & 127.02 & 98.47 \\
\hline $1995-1999$ & 68.35 & 125.67 & 97.01 \\
\hline $2000-2004$ & 61.05 & 113.18 & 87.12 \\
\hline $2005-2009$ & 47.72 & 98.97 & 73.35 \\
\hline $2010-2014$ & 38.41 & 85.91 & 62.17 \\
\hline
\end{tabular}

Elaboración propia con datos de anuarios estadísticos y del Centro Centroamericano de Población.

a Âiajueia y Cararago pasó ai segundo iugar, en tanto que San José ha tenido la tasa más alta a lo largo de los últimos treinta años. La tasa más baja le correspondió a la
Tabla 5. Coeficiente de regresión ( $r$ ) y de determinación $\left(r^{2}\right)$, coeficiente $\beta$ y significancia (p) para las tasas ajustadas de mortalidad por causa de cardiopatía isquémica coronaria en personas de 35 a 74 años, según sexo. Costa Rica 1970-2014

\begin{tabular}{|l|c|c|c|c|}
\hline Sexo y total & $\mathbf{r}$ & $\mathbf{r}^{2}$ & $\beta$ & $\mathbf{p}$ \\
\hline 1970-1999 & & & & \\
\hline Hombres & 0.6530 & 0.4264 & 1.1389 & 0.05 \\
\hline Mujeres & 0.0677 & 0.0046 & 0.1269 & 0.62 \\
\hline Total & 0.5354 & 0.2867 & 0.6329 & 0.10 \\
\hline $2000-2014$ & & & & \\
\hline Hombres & 0.9994 & 0.9988 & -2.7270 & 0.02 \\
\hline Mujeres & 0.9896 & 0.9793 & -2.2640 & 0.07 \\
\hline Total & 0.9964 & 0.9928 & -2.4950 & 0.04 \\
\hline
\end{tabular}

provincia de Guanacaste, aunque en los últimos quinquenios las tasas de Puntarenas y Limón se le han acercado mucho, hasta casi igualarse (Tabla 6). 


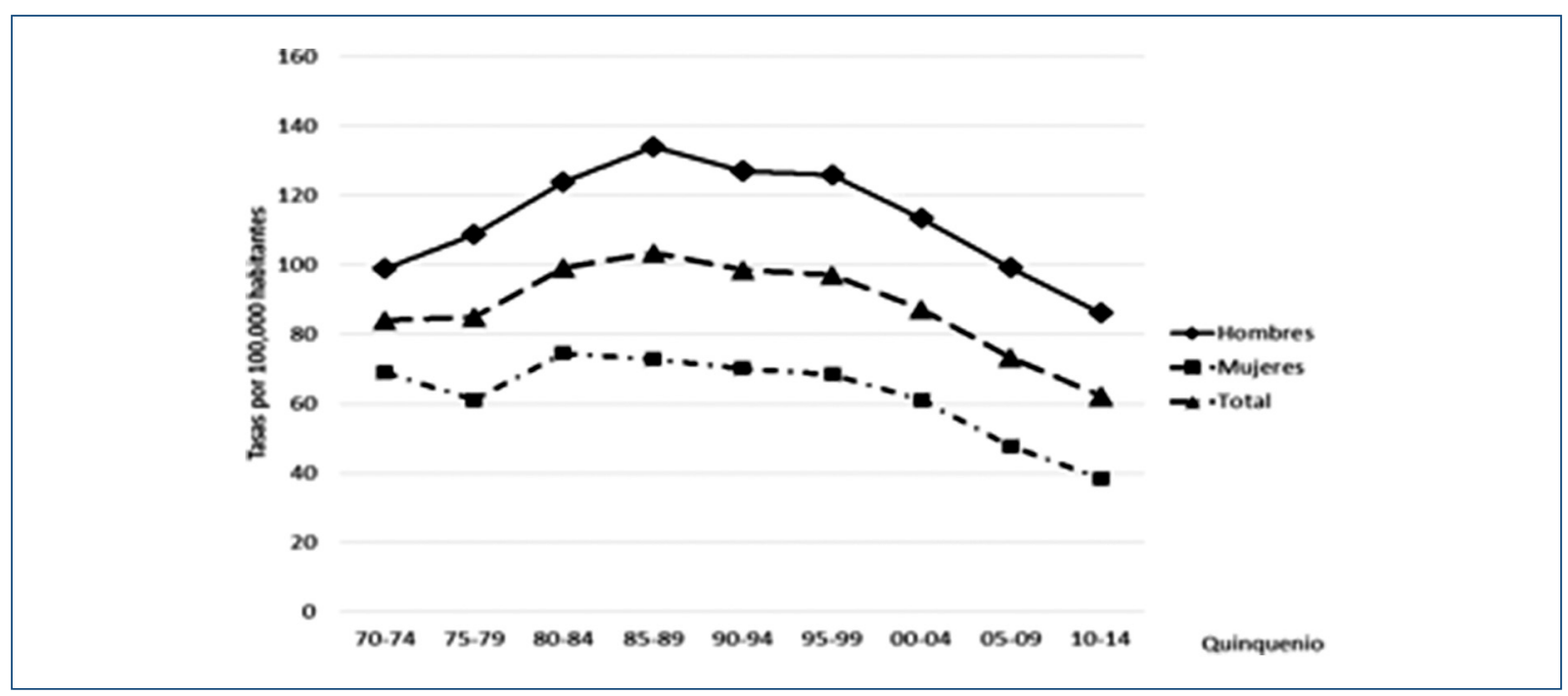

Figura 2. Tasas ajustadas de mortalidad por enfermedad isquémica del corazón en personas de 35 a 74 años, según sexo. Costa Rica, 1970-214 (elaborada a partir de datos de anuarios estadísticos y del Centro Centroamericano de Población).

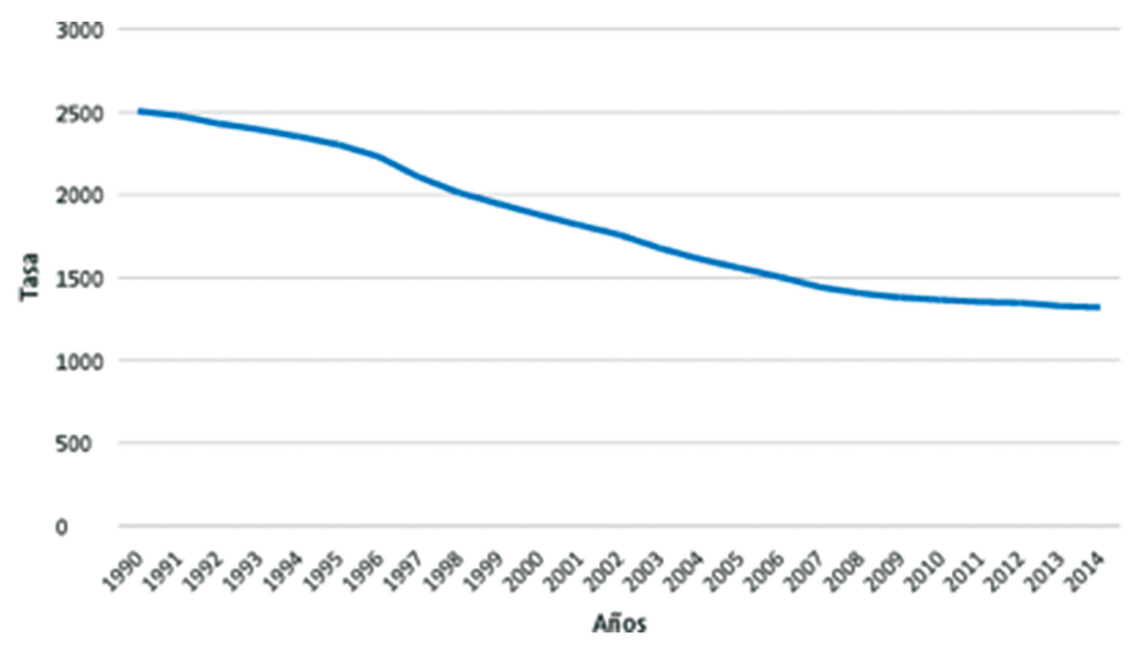

Figura 3. Años de vida perdidos ajustados por discapacidad por enfermedad isquémica del corazón en Costa Rica, periodo 1990-2014. Tasas por 100,000 habitantes (elaborada a partir de datos del Institute for Health Metrics and Evaluation $\left.{ }^{20}\right)$.

Las tasas estandarizadas por provincias han tenido un comportamiento bastante semejante al de las tasas brutas señaladas anteriormente (Tabla 6).

Para la distribución geográfica de la mortalidad por EIC también recurrimos al análisis por regiones socioeconómicas de Costa Rica, igualmente tomando en cuenta tasas brutas y estandarizadas. Las mismas se aprecian en las la tabla 7
En el caso de las tasas brutas, las más altas correspondieron a la región Central, seguida por la Huétar Atlántica. Las tasas más bajas se observaron en las regiones de Chorotega y Huétar Norte.

Con relación a la evolución de las tasas estandarizadas, fue bastante semejante a la que tuvieron las tasas brutas, siendo las más elevadas las de la región central (con excepción de los dos primeros quinquenios, en 


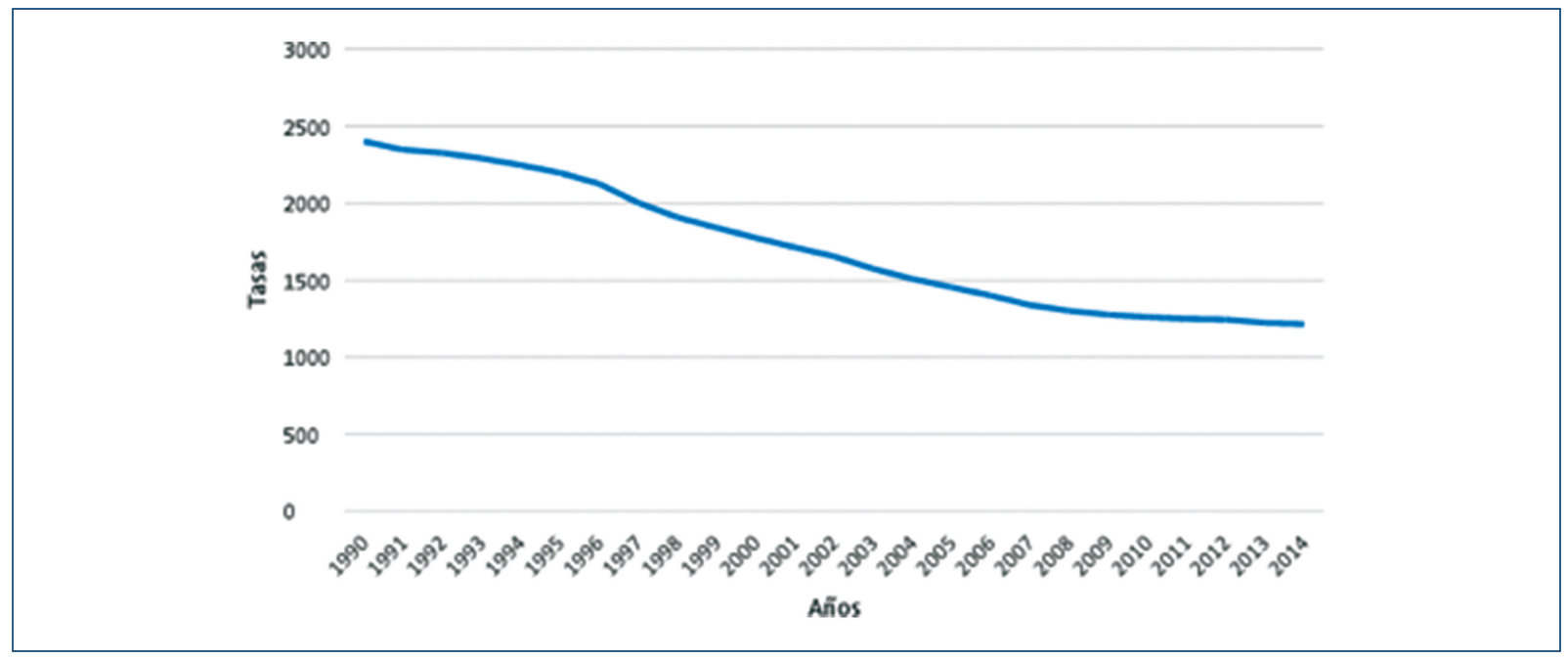

Figura 4. Años de vida potencialmente perdidos por enfermedad isquémica del corazón en Costa Rica, periodo 1990-2014. Tasas por 100,000 habitantes (elaborada a partir de datos del Institute for Health Metrics and Evaluation ${ }^{20}$ ).

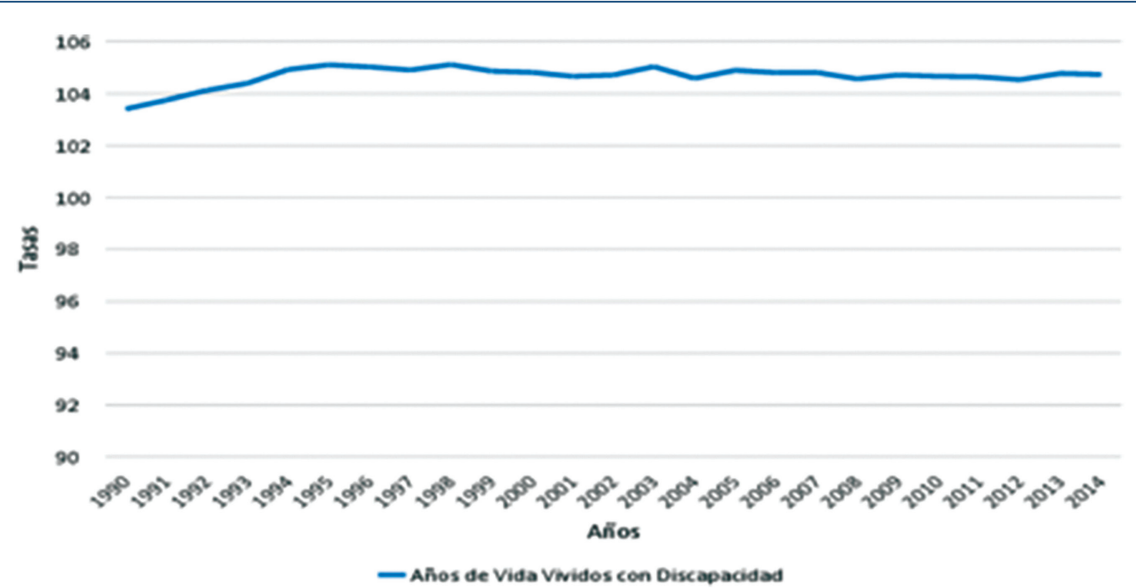

Figura 5. Años de vida con discapacidad por enfermedad isquémica del corazón en Costa Rica, periodo 1990-2014. Tasas por 100,000 habitantes (elaborada a partir de datos del Institute for Health Metrics and Evaluation ${ }^{20}$ ).

que fueron superadas por las tasas de la región Huétar Atlántica, que en el resto del periodo obtuvieron el segundo lugar), correspondiéndoles las más bajas a las regiones de Chorotega y Huétar Norte.

\section{Discusión}

La EIC representó el 10.9\% de la mortalidad en América Latina y el Caribe, ocupando el primer lugar como causa específica de muerte ${ }^{6}$, así como también ${ }^{\circ}$ fue en el resto del mundo ${ }^{21}$. Las tasas brutas de mortalidad por esta causa en Costa Rica son ligeramente inferiores al promedio para las Américas y en particular, para América Latina, tanto para hombres y mujeres como para ambos sexos (ver Tabla 1). También lo son en el istmo centroamericano, dado que Guatemala, Nicaragua y Panamá tienen tasas más elevadas, siendo las de Costa Rica casi iguales a las del Salvador y Belice, pero superando claramente a la de Honduras. Dichas tasas brutas mostraron una clara tendencia al aumento durante gran parte del periodo estudiado, salvo en los años finales, en los que se pudo apreciar un descenso que solamente se interrumpió en el quinquenio 2010-2014. 
Tabla 6. Tasas brutas y estandarizadas de mortalidad por causa de Enfermedad Isquémica del Corazón en personas de 35 a 74 años, por quinquenio según provincia. Costa Rica 1970-2014

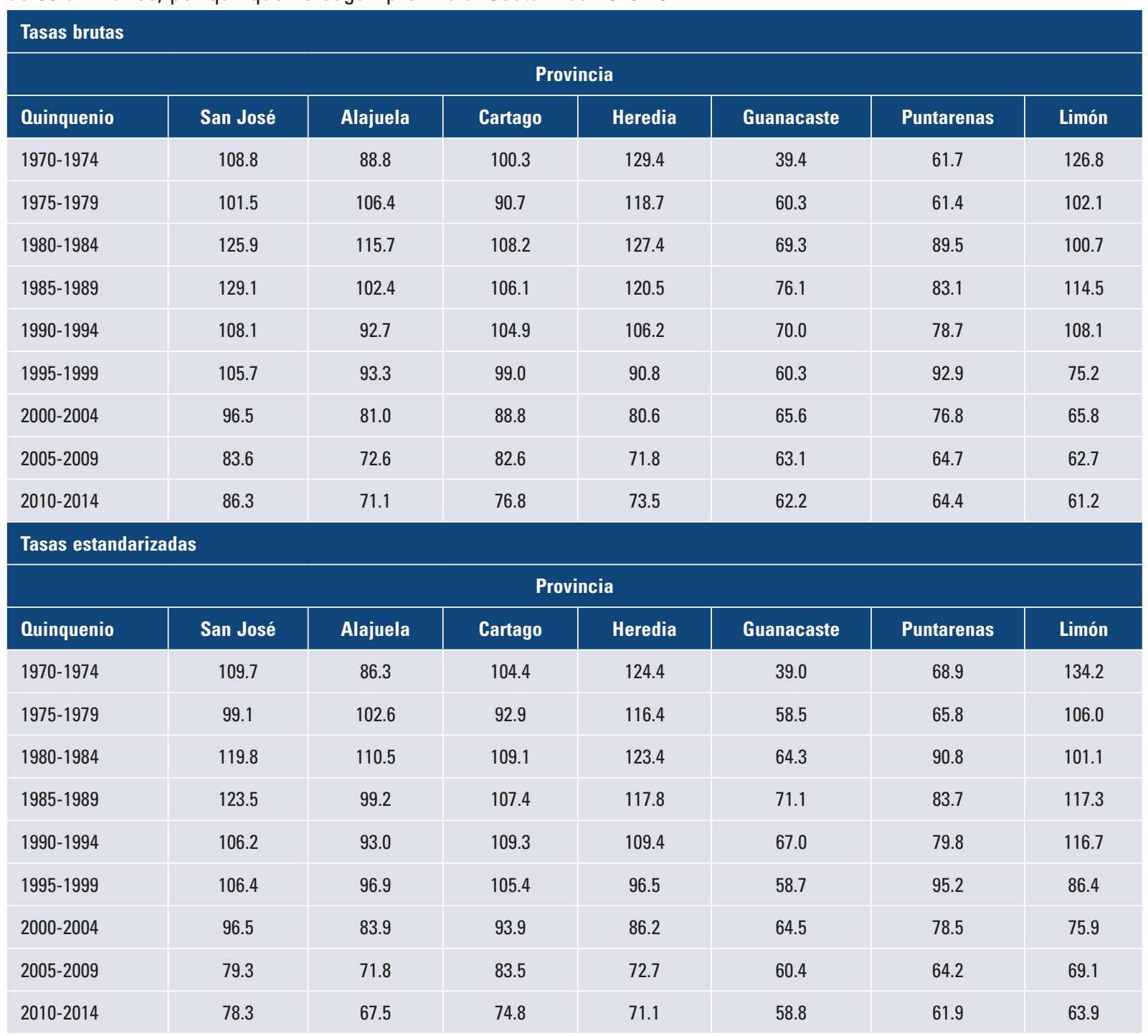

Elaboradas a partir de datos del Instituto Nacional de Estadística y el Centro Centroamericano de Población

En un estudio previo realizado por otros autores ${ }^{i \hat{}} \mathrm{en}$ relacióncon la mortalidad por EIC durante el periodo 1970-2002 se encontró que la mortalidad aumentó en un $18.4 \%$, correspondiendo un $6.1 \%$ a las mujeres y un $28.4 \%$ a los varones. En nuestra investigación también se aprecia un aumento de las tasas brutas de EIC hasta el año 2002, a partir del cual comienza un leve descenso que continúa en una meseta hasta el año 2009, para ser seguida por un aumento entre los años 2010 y 2014. Lo anterior se observa mejor cuando presentamos la información por quinquenios (Tabla 3), cuando la tendencia al descenso durante 2000-2009 es interrumpida de manera brusca en los siguientes cinco años. Postulamos que quizás esta conducta se debe a que Costa
Rica en los últimos quinquenios ha aumentado significativamente la expectativa de vida, permitiendo así que un mayor número de personas estén expuestas a morir por EIC. Se trata en cierto sentido de una emigración de la anterior mortalidad a las edades mayores de la vida. También puede mencionarse el aumento desmedido de la obesidad y el sobrepeso en Costa Rica.

En cuanto a tasas estandarizadas, se obtuvieron las del grupo etario de 35 a 74 años, por ser este el que detenta la probabilidad mayor de presentar o de morir por un evento coronario. En este sentido, se observa que las tasas se incrementaron continuamente hasta el quinquenio 1985-89, a partir del cual la tendencia fue hacia el descenso franco, tal como puede observarse 
Tabla 7. Tasas brutas y estandarizadas de mortalidad por causa de enfermedad isquémica del corazón en personas de 35 a 74 años, por quinquenio según región socioeconómica. Costa Rica 1970-2014

\begin{tabular}{|c|c|c|c|c|c|c|}
\hline Tasas brutas & & & Región so & & \multicolumn{2}{|c|}{ Región socioeconómica } \\
\hline Quinquenio & Central & Chorotega & P Central & Brunca & H Atlántica & H Norte \\
\hline $1970-1974$ & 109.3 & 40.6 & 71.7 & 54.8 & 126.8 & 48.1 \\
\hline $1975-1979$ & 107.9 & 57.2 & 81.5 & 50.4 & 102.1 & 56.6 \\
\hline $1980-1984$ & 127.2 & 67.4 & 94.0 & 78.3 & 100.7 & 75.4 \\
\hline 1985-1989 & 124.1 & 73.5 & 97.5 & 75.4 & 114.5 & 67.9 \\
\hline 1990-1994 & 108.7 & 69.7 & 89.8 & 67.0 & 108.1 & 59.8 \\
\hline 1995-1999 & 103.1 & 61.3 & 101.7 & 81.8 & 75.2 & 69.9 \\
\hline $2000-2004$ & 92.4 & 63.7 & 82.9 & 73.1 & 65.8 & 64.8 \\
\hline $2005-2009$ & 81.8 & 62.1 & 75.0 & 59.6 & 62.7 & 54.8 \\
\hline $2010-2014$ & 81.7 & 60.9 & 70.8 & 58.5 & 61.2 & 63.3 \\
\hline \multicolumn{7}{|c|}{ Tasas estandarizadas } \\
\hline \multicolumn{7}{|c|}{ Región socioeconómica } \\
\hline Quinquenio & Central & Chorotega & P Central & Brunca & H Atlántica & H Norte \\
\hline 1970-1974 & 108.2 & 40.3 & 72.5 & 67.6 & 134.2 & 52.5 \\
\hline 1975-1979 & 104.6 & 56.2 & 81.3 & 56.2 & 106.0 & 62.9 \\
\hline $1980-1984$ & 121.2 & 63.6 & 89.8 & 81.5 & 101.1 & 84.1 \\
\hline 1985-1989 & 119.3 & 69.5 & 92.9 & 77.8 & 117.3 & 74.8 \\
\hline 1990-1994 & 108.2 & 67.2 & 88.4 & 69.1 & 116.7 & 67.0 \\
\hline 1995-1999 & 105.6 & 59.6 & 103.2 & 84.3 & 86.4 & 78.7 \\
\hline $2000-2004$ & 94.3 & 62.5 & 84.4 & 74.5 & 75.9 & 71.8 \\
\hline $2005-2009$ & 79.2 & 59.5 & 73.6 & 59.1 & 69.1 & 58.7 \\
\hline $2010-2014$ & 75.8 & 57.6 & 66.9 & 56.6 & 63.9 & 65.6 \\
\hline
\end{tabular}

en la tabla 4 y en la figura 2. Este descenso en nuestro estudio fue estadísticamente significativo (Tabla 5) para ambos sexos $(p=0.04)$ y para los varones $(p=00.2)$, pero no para las mujeres $(p=0.07)$. El descenso porcentual entre ambos periodos extremos fue equivalente al $39.8 \%$. En este caso se observa una tendencia semejante a la que han tenido los países desarrollados ${ }^{22}$, salvo que el descenso de las tasas estandarizadas por edad se inició en ellos mucho antes, a partir de 1980, como consecuencia del éxito de la prevención cardiovascular primaria y secundaria ${ }^{21}$.No obstante ese gran descenso de la EIC que ha sucedido en las naciones desarrolladas, todavía en ellas son la causa de una de cada tres muertes en la población mayor de 35 años ${ }^{23}$.

La información anterior nos permite conocer que en todos los quinquenios comprendidos entre el de
1970-1974 hasta el de 2010-2014 las tasas de EIC fueron superiores en los hombres con respecto a las mujeres, siendo la razón menor al principio de la serie (1.43:1) y la razón mayor al final de esta (2.23:1). Se observa además que la razón favorable a los varones se fue incrementando a lo largo de todos los quinquenios, resultado que coincide con otro estudio realizado en el país ${ }^{11}$, aparte de resultados de otras naciones, como Méxi$\mathrm{CO}^{24,25}$. La expectativa de vida en Costa Rica siempre ha sido mayor en las mujeres en comparación de los hombres (82.2 años para las mujeres y 77.5 para los hombres, año 2016), lo que explicaría en parte la anterior característica. También se ha escrito que la mayor mortalidad en los varones debido a EIC podría deberse a las tasas más elevadas de fumadores (18.8\% en varones y $7.9 \%$ en mujeres, para el año 2014) y de consumidores 
de bebidas alcohólicas (varones $44.4 \%$ y mujeres $26.8 \%$ durante el mismo año). En este último caso se refiere a personas que dijeron haber consumido en los últimos doce meses al menos una bebida alcohólica ${ }^{26}$. En igual sentido, la misma segunda encuesta de factores de riesgo cardiovasculares señala que "se obtuvo una prevalencia del $41.7 \%$ de actividad física alta en las personas del sexo masculino, siendo mayor en el grupo de 20 a 39 años (48\%), mientras que en el sexo femenino la prevalencia de actividad física alta fue del $26.2 \%$, siendo mayor en el grupo de 40 a 64 años $(28.9 \%$ ) »26.

Otros de los más importantes factores de riesgo reconocidos por múltiples investigaciones epidemiológicas son el sobrepeso y la obesidad. Precisamente ambas condiciones han empeorado mucho en Costa Rica. Así, por ejemplo, el sobrepeso para ambos sexos pasó del $24.9 \%$ en 1975 al $36.1 \%$ en el 2014 , para un aumento porcentual del $44.90 \%$, con la particularidad de que los varones tuvieron prevalencias mayores que las mujeres, dado que del $24.5 \%$ en 1975 pasaron al $39.3 \%$ en el 2014 , mientras que las mujeres variaron del $25.4 \%$ en el primero de los años mencionados al $33.0 \%$ en el último. En el caso de la obesidad (igual o mayor de $30 \mathrm{~kg} / \mathrm{m}^{2}$ ) se pasó del $6.3 \%$ en 1975 al $23.7 \%$ en el 2014. En este caso, las mujeres tuvieron prevalencias mayores, ya que del 8.5 se aumentó al $28.5 \%$, en tanto los varones variaron del 7.8 al $19.0 \%{ }^{27}$. Podría postularse que el aumento de la mortalidad observado en la mortalidad por EIC durante el último quinquenio (2010-2014) estaría relacionado en buena medida a este aumento de la obesidad y el sobrepeso en Costa Rica. En otros países también se ha mencionado este efecto negativo de ambas condiciones, así como de la diabetes, sobre la mortalidad por las ECV, mermando la posibilidad del descenso continuo de sus tasas.

Además, se obtuvieron otros indicadores que no solamente miden la mortalidad sino también la morbilidad, expresada en este caso por la discapacidad. La medición de la carga de la enfermedad ha sido uno de los acontecimientos cumbre de la epidemiología de los años recientes, especialmente cuando a partir de los años noventa, por iniciativa del Banco Mundial, se presentó el primer informe sobre dicha materia ${ }^{28}$. De allí surgieron los AVAD, que lograron conjuntar las consecuencias mortales y no mortales de las enfermedades, lesiones y factores de riesgo,representadas por el indicador AVP y por los AVD. Cuando se analizan las figuras 4 (AVP) y la 5 (AVD) se encuentra que los AVAD están constituidos en más de un $90 \%$ por los AVP, correspondiéndole un porcentaje menor al $10 \%$ a los AVD, situación bastante parecida a la ocurrida en España, en donde un $96 \%$ fue aportado por los años de vida perdidos por muerte prematura y apenas un $4 \%$ por discapacidad ${ }^{29}$; parecidos resultados se han notificado en Colombia ${ }^{30}$. Tanto la suma de los AVAD como los AVP tienen tendencia a disminuir, estos últimos pasaron de tener una tasa de 13,705 por cien mil habitantes en el año 1990 a 10,447 en el 2010. En cambio, los AVD prácticamente no experimentaron cambios significativos, puesto que de una tasa estandarizada de 11,672 por cien mil habitantes en 1990 pasaron a una de 10,948 en el $2010^{31}$.

De acuerdo con una publicación de la Caja Costarricense de Seguro Social ${ }^{32}$, basada en información aportada por el IHME, Ios AVP por EIC pasaron de ser la segunda causa de muerte específica en 1990 a constituir la primera en el 2010. También en esa misma publicación, las EIC fueron la primera causa de AVAD en Costa Rica, mientras que como causa de AVD su aporte fue prácticamente insignificante, dado que ocuparon el puesto 25.

El aporte porcentual de la EIC a la mortalidad general igualmente se incrementó con el tiempo, pero a partir del quinquenio 1985-89 el porcentaje se estabilizó alrededor de un 14\%, bastante superior al de América Latina, como se mencionó líneas atrás.

En lo que se refiere a la distribución porcentual de los componentes específicos de la EIC tenemos que en nuestro estudio el IAM ocupó el puesto principal, aunque con variaciones importantes que han oscilado entre el 55 y el $70 \%$. En una investigación realizada en México los porcentajes encontrados fueron del $81.24 \%$ en el 2001 y del $82.75 \%$ en el año $2002^{25}$.

\section{Conclusiones}

En la evolución de la mortalidad por EIC en Costa Rica pueden identificarse tres etapas bastante nítidas. La primera se extiende de 1930 al año 1964, caracterizada por un ascenso lento pero continuo, al que sigue un aumento explosivo de las tasas, que llega al año 1984. Seguidamente las tasas tienen tendencia a la estabilización y al descenso, que se ve interrumpido en el último quinquenio.

Cuando se toma en cuenta la mortalidad del grupo etario de 35 a 74 años por quinquenios, en este caso durante el periodo 1970-1974, 2010-2014, las tasas estandarizadas permiten identificar dos periodos muy nítidos. El primero, que va del inicio hasta el quinquenio 1985-1989, se caracteriza por un incremento continuo de las tasas, que es seguido por un descenso ininterrumpido hasta el quinquenio final del lapso de tiempo. En este caso, a diferencia de la mortalidad por 
EIC de toda la población, la franca tendencia hacia la baja no se detiene al final de la serie.

\section{Conflicto de intereses}

Los autores declaran no tener conflicto de intereses ni haber recibido recibido ayudas financieras específicas provenientes de agencias del sector público, sector comercial o entidades sin ánimo de lucro.

\section{Responsabilidades éticas}

\section{Protección de personas y animales}

Los autores declaran que para esta investigación no se han realizado experimentos en seres humanos ni en animales.

\section{Confidencialidad de los datos}

Los autores declaran que han seguido los protocolos de su centro de trabajo sobre la publicación de datos de pacientes.

\section{Derecho a la privacidad y consentimiento informado}

Los autores declaran que en este artículo no aparecen datos de pacientes.

\section{Bibliografía}

1. Mozaffarian D, Benjamin EJ, Go AS, Arnett DK, Blaha MJ, Cushman M, et al. Heart Disease and Stroke Statistics-2016 Update: A report from the American Heart Association. Circulation. 2016;133(4):e38-360.

2. Sagar B. Dugani Andrew E. Moran Robert O. Bonow Thomas A. Gaziano. Ischemic heart disease: Cost-Effective acute management and secondary prevention. En: Prabhakaran D, Anand S, Gaziano TA, Mbanya JC, Wu Y, Nugent R, editores. Disease Control Priorities, Third Edition (Volume 5): Cardiovascular, Respiratory, and Related Disorders [Internet]. The World Bank Group; 2017 [fecha de publicación: noviembre de 2017]. Disponible en: https://elibrary.worldbank.org/doi/ abs/10.1596/978-1-4648-0518-9 ch8

3. Hay SI, Abajobir AA, Abate KH, Abbafati C, Abbas KM, Abd-Allah F, et al. Global, regional, and national disability-adjusted life-years (DALYs) for 333 diseases and injuries and healthy life expectancy (HALE) for 195 countries and territories, 1990-2016: a systematic analysis for the Global Burden of Disease Study 2016. Lancet. 2017;390(10100):1260-344.

4. Naghavi M, Abajobir AA, Abbafati C, Abbas KM, Abd-Allah F, Abera SF et al. Global, regional, and national age-sex specific mortality for 264 causes of death, 1980-2016: a systematic analysis for the Global Burden of Disease Study 2016. Lancet. 2017;390(10100):1151-210.

5. Health at a Glance 2017 [Internet]. Organisation for Economic Co-operation and Development. OECD Indicators [citado 19 de abril de 2018] Disponible en: http://www.oecd.org/health/health-systems/health-at-aglance-19991312.htm

6. Situación de Salud en las Américas: Indicadores Básicos 2016 [Internet] Organización Panamericana de la Salud. Organización Mundial de la Salud [citado: 19 de abril de 2018]. Disponible en: http://iris.paho.org/ xmlui/handle/123456789/31288
7. Evans-Meza R, Pérez-Fallas J, Bonilla-Carrión R. Análisis de la mortalidad por enfermedades cerebrovasculares en Costa Rica entre los años 1920-2009. Arch Cardiol Mex. 2016;86(4):358-66.

8. Vos T, Abajobir AA, Abate KH, Abbafati C, Abbas KM, Abd-Allah F, et al. Global, regional, and national incidence, prevalence, and years lived with disability for 328 diseases and injuries for 195 countries, 1990-2016: a systematic analysis for the Global Burden of Disease Study 2016. Lancet. 2017:390(10100):1211-59.

9. Memoria Institucional 2016. [Internet]. [citado 19 de abril de 2018]. Disponible en: https://www.ministeriodesalud.go.cr/images/stories/docs/ DPI/2017/DPI memoria_institucional_2016.pdf

10. Roselló Araya M, Guzmán Padilla $\bar{S}$. Mortalidad por infarto agudo de miocardio: distribución geográfica y lugar de ocurrencia. Costa Rica, 1970-2002. Rev Costarric Cardiol. 2003:5(3):25-9.

11. Roselló Araya M, Guzmán Padilla S. Evolución de la mortalidad por enfermedad isquémica del corazón e infarto agudo del miocardio en Costa Rica, 1970-2001. Rev Panam Salud Publica. 2004;16:295-301.

12. Provincias de Costa Rica y sus límites I Temarios y Tareas [Internet] [citado 22 de octubre de 2018]. Disponible en: https://temariosytareas. wordpress.com/2010/03/11/provincias-de-costa-rica/

13. Instituto Nacional Estadística y Censos. Anuarios estadísticos 19201969. San José, Costa Rica.

14. Estadísticas vitales de mortalidad. Costa Rica 1970-2014 [Internet]. Centro Centroamericano de Población [citado: 19 de abril de 2018]. Disponible en: http://ccp.ucr.ac.cr.

15. Camel-Vargas F. Estadísticas médicas y de salud pública. Universidad de Los Andes, Mérida (Venezuela). Talleres Gráficos Universitarios; 1966.

16. Boletín Demográfico $\mathrm{N} .{ }^{\circ} 73$. América Latina y el Caribe: estimaciones y proyecciones de población. 1950-2050. Santiago de Chile: CELADE. División de Población; 2004.

17. Moran A, Odden MC. Tendencias de la mortalidad por infarto de miocardio en España y Estados Unidos: ¿una carrera cuesta abajo o cuesta arriba en el siglo XXI? Rev Esp Cardiol. 2012;65(12):1069-71.

18. Stata Corp 2014.Stata Statistical Sofware: Release 14 College Station, TX: Stata Corp LP.

19. Lozano R, Murray CJL, López AD, Satoh T. Miscoding and misclassification of ischemic heart disease mortality. Global Programme on Evidence for Health Policy Working Paper No. 12 [Internet]. World Health Organization; September 2001. Disponible en: http://www.who. int/ healthinfo/paper12.pdf

20. GBD Compare I IHME Viz Hub [Internet]. University of Washington: Institute for Health Metrics and Evaluation [citado: 16 de noviembre de 2017]. Disponible en: http://vizhub.healthdata.org/gbd-compare

21. Moran AE, Forouzanfar MH, Roth GA, Mensah GA, Ezzati M, Flaxman A, et al. The Global Burden of Ischemic Heart Disease in 1990 and 2010 The Global Burden of Disease 2010 Study. Circulation. 2014;129(14): 1493-501.

22. Sanchis-Gomar F, Perez-Quilis C, Leischik R, Lucia A. Epidemiology of coronary heart disease and acute coronary syndrome. Ann Transl Med. 2016;4(13):256.

23. Bhatnagar P, Wickramasinghe K, Wilkins E, Townsend N. Trends in the epidemiology of cardiovascular disease in the UK. Heart. 2016;102(24):1945-52.

24. Lozano R, Escamilla JA, Escobedo J, López M. Tendencia de la mortalidad por cardiopatía isquémica en México, de 1950 a 1985. Salud Pública de México. 1990;32(4):405-15.

25. Velázquez Monroy O, Barinagarrementería Aldatz FS, Rubio Guerra AF, Verdejo J, Méndez Bello MÁ, Violante R, et al. Morbilidad y mortalidad de la enfermedad isquémica del corazón y cerebrovascular en México. 2005. Arch Cardiol Méx. 2007;77(1):31-9.

26. Encuesta de Factores de Riesgo de ECNT. CCSS. Vigilancia de los factores de riesgo cardiovasculares. Segunda encuesta, 2014. Roy Wong McClure, editor. San José, Costa Rica. EDNASSS-CCSS.

27. Evans-Meza R, Pérez-Fallas J. Tendencias del índice de masa corporal en Costa Rica de 1975 al 2014. Rev Hisp Cienc Salud. 2016;2(2):153-60.

28. GBD History [Internet]. Institute for Health Metrics and Evaluation, University of Washington [citado 19 de abril de 2018]. Disponible en: http:// www.healthdata.org/about/history

29. Fernández de Larrea-Baz N, Morant-Ginestar C, Catalá-López F, Gènova-Maleras R, Álvarez-Martín E. Disability-adjusted life years lost to ischemic heart disease in Spain. Rev Esp Cardiol. 2015;68:968-75.

30. Bolívar-Mejía A, Vesga-Angarita BE. Burden of cardiovascular disease in Colombia. Current Topics in Public Health, Alfonso J. Rodriguez-Morales [Internet]. Colombia; 2013 [citado 23 de abril 2018]. IntechOpen. DOI: 10.5772/53280. Disponible en: https://www.intechopen.com/books/current-topics-in-public-health/burden-of-cardiovascular-disease-in-colombia

31. Costa Rica Profile [Internet]. Institute for Health Metrics and Evaluation, University of Washington [citado: 23 de abril de 2018]. Disponible en: http://www.healthdata.org/costa-rica

32. La carga de enfermedad y esperanza de vida saludable-Costa Rica. 2014. Caja Costarricense de Seguro Social. San José, Costa Rica; 2014 